
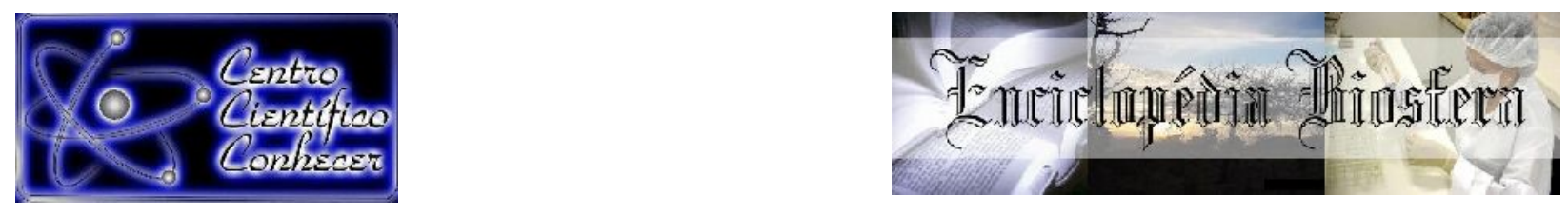

\title{
AVALIAÇÃO E COMPARAÇÃO DA QUALIDADE DE VIDA DE ADOLESCENTES INGRESSANTES EM UM PROJETO MULTIPROFISSIONAL COM FOCO NO TRATAMENTO DO EXCESSO DE PESO OU OBESIDADE
}

Alexandre Pereira Reis ${ }^{1}$; Natalia Quevedo dos Santos ${ }^{2}$; Fabiano Mendes de Oliveira $^{3}$; Sônia Maria Marques Gomes Bertolini ${ }^{4}$; Braulio Henrique Magnani Branco ${ }^{5}$

\author{
${ }^{1}$ Graduado em Educação Física, Centro Universitário de Maringá (UniCesumar), \\ Maringá - PR, Brasil. \\ ${ }^{2}$ Graduada em Fisioterapia, Mestranda no Programa de Pós-Graduação Promoção \\ da Saúde da UniCesumar, bolsista (CAPES), Maringá - PR, Brasil. E-mail: \\ natquevedo01@gmail.com \\ ${ }^{3}$ Graduado em Educação Física da UniCesumar, Maringá - PR, Brasil. \\ ${ }^{4}$ Doutora em Morfologia Humana, Docente do Programa de Pós-Graduação em \\ Promoção da Saúde e Pesquisadora do Instituto Cesumar de Ciência, Tecnologia e \\ Inovação (ICETI), Maringá - PR, Brasil. \\ ${ }^{5}$ Doutor em Biodinâmica do Movimento Humano, Docente do Programa de Pós- \\ Graduação em Promoção da Saúde e Pesquisador do ICETI, Maringá - PR, Brasil.
}

\section{Recebido em: 06/04/2019 - Aprovado em: 10/06/2019 - Publicado em: 30/06/2019 DOI: 10.18677/EnciBio_2019A155}

\begin{abstract}
A obesidade se tornou nas últimas décadas um problema de saúde pública em nível mundial, acometendo diferentes faixas etárias com prejuízos físicos, emocionais e sociais. Diante disso, os adolescentes têm sido grandemente impactados por essa doença crônica não-transmissível, que é considerada nos dias atuais, como a pandemia do século XXI. Nesse sentido, o presente estudo teve como objetivo avaliar e comparar por sexo, a qualidade de vida de adolescentes com excesso de peso ou obesidade. Para tanto, utilizou-se o inventário de avaliação pediátrica "pediatric quality of life inventory" (PedsQL4.0 TM $)$. No referido instrumento, foram analisados os seguintes aspectos: qualidade de vida dos adolescentes, divididos em 4 dimensões: problemas quanto à saúde, aos sentimentos, relacionamentos e escola. Quanto aos resultados, foram verificados baixos índices de qualidade de vida para os diferentes domínios analisados (físico: $6,1 \pm 4,1$; emocional: 7,0 $\pm 4,2$; social: 3,9 $\pm 3,3$ e escolar: 5,5 $\pm 3,5)$. Além disso, não foram identificadas diferenças significativas em relação ao sexo, para os quatro domínios mensurados $(p>0,05)$. Com base nos resultados apresentados, conclui-se que a percepção da qualidade de vida é baixa e sem diferenças em relação ao sexo dos adolescentes.
\end{abstract}

PALAVRAS-CHAVE: atenção à saúde, equidade em saúde, prevenção de doenças, saúde do adolescente. 


\title{
EVALUATION AND COMPARISON OF THE QUALITY OF LIFE OF ADOLESCENTS ENTRANTS IN A MULTIPROFESSIONAL PROJECT WITH A FOCUS ON THE TREATMENT OF OVERWEIGHT OR OBESITY
}

\begin{abstract}
In the last decades, the obesity has become a public health problem worldwide, affecting different age groups with physical, emotional and social damages. In light of this, adolescents have been greatly impacted by this chronic non-communicable disease, which is considered today as the $21^{\text {st }}$ century pandemic. For that matter, the present study aimed to evaluate and to compare by gender the quality of life of adolescents with overweight or obesity. The Pediatric Quality of Life Inventory (PedsQL4.0 TM) was used for this purpose. In this instrument, the following aspects were analyzed: quality of life of adolescents, divided into 4 dimensions: problems regarding health, feelings, relationships and school. In relation to the results, low quality of life indices were verified for the different domains analyzed (physical: $6.1 \pm$ 4.1, emotional: $7.0 \pm 4.2$, social: $3.9 \pm 3.3$ and scholar: $5.5 \pm 3.5$ ). Furthermore, no significant gender differences were identified for the four domains measured $(p>$ $0.05)$. Based on the results presented, it is concluded that the perception of quality of life is low and without differences in relation to the gender of the adolescents.
\end{abstract}

KEYWORDS: health care, health equity, disease prevention, adolescent health.

\section{INTRODUÇÃO}

A Organização Mundial da Saúde definiu qualidade de vida como a percepção do indivíduo de sua respectiva posição na vida, dentro do contexto: cultura e sistema de valores nos quais vivenciados, tendo em vista os objetivos, expectativas, padrões e preocupações (WHO, 2018). A definição salienta a relevância da avaliação da qualidade de vida, com caráter multidimensional, englobando múltiplos aspectos: a) aspecto físico, b) funcional, c) além do bem-estar psicológico e social (SOUZA et. al., 2014).

A avaliação da qualidade de vida é um importante indicador de saúde e exige que o profissional de saúde não analise exclusivamente os aspectos biológicos concatenados à obesidade, devendo-se, deste modo, considerar também a abordagem psicossocial (PANTOJA et al., 2017). Em consequência, na década de 1990, consubstanciou-se a ideia de que os instrumentos de avaliação da qualidade de vida devem considerar as percepções das pessoas e não se limitar apenas às repostas das perspectivas dos profissionais de saúde, assim como da comunidade acadêmica (LA SCALA et al., 2005).

A princípio, a avaliação da qualidade de vida realizou-se pela percepção dos pais, haja visto que as crianças seriam incapazes de preencher semelhante tarefa. Ainda assim, preliminares esclarecimentos permitiram ratificar a capacidade psicológica do grupo populacional a fim de avaliar e compreender questões relacionadas à vida (PEROSA et al., 2013).

Os modelos mais recentes sustentam uma abordagem modular e integrada, em que se associam medidas mais genéricas, utilizando parâmetros mais específicos para avaliação da saúde em diferentes esferas (CUNHA et al., 2018). Apesar disso, existem pontos de vista que tem norteado o desenvolvimento do conceito de saúde na adolescência, na qual, destacam-se: a auto percepção da saúde e qualidade de vida em diferentes âmbitos, como por exemplo: físico, emocional, social e escolar (NARDO-JÚNIOR et al., 2018). À vista disso, a inclusão das crianças como colaboradores de pesquisas depende da apta capacidade em 
responder questões estabelecidas, respeitando permanentemente os limites, no processo complexo de qualidade de vida relacionado à saúde (QVRS). Sob esta dimensão, estudos que concernem QVRS em indivíduos com excesso de peso ou obesidade, estão se tornando cada vez mais habituais (KOLOTKIN; ANDERSEN, 2017). Diante do cenário, corrobora-se um aumento exponencial na prevalência de excesso de peso ou obesidade na infância e adolescência.

Adicionalmente, despertou-se elevado interesse em estudar as consequências psicossociais dos agravos relacionados ao excesso de peso e sobretudo, a obesidade na adolescência (ROCHA et al., 2017). Portanto, verifica-se que a obesidade pode impactar diretamente aspectos biopsicossociais nessa faixa etária da população (BRANCO et al., 2018). Neste ensejo, os momentos mais críticos para o aparecimento da obesidade relacionam-se com a transição da infância para a adolescência (MULLER, 2001). Etapa na qual o adolescente apresenta incessante preocupação com peso corporal, visando um ideal de corpo magro, imposto pela mídia e em vista disso, ocorrem por vezes a insatisfação com a imagem corporal, na ocasião em que os jovens denotam excesso de peso ou obesidade (GOUVEIA et al., 2014; SILVA et al., 2017). Em virtude disso, a nãoaceitação com seu próprio corpo, pode levá-lo a se sentir marginalizado no meio social (BRANCO et al., 2006).

Destarte, considerando os aspectos supra elencados, os objetivos do presente estudo foram: avaliar e comparar por sexo, a qualidade de vida de adolescentes ingressantes em um projeto multiprofissional com foco no tratamento do excesso de peso ou obesidade.

\section{Delineamento da pesquisa}

\section{MATERIAL E MÉTODOS}

O presente estudo foi aprovado pelo comitê de ética e pesquisa local, sob o número: 2.505.200/2018 do centro Universitário de Maringá. As coletas foram realizadas no Laboratório Interdisciplinar de Intervenção em Promoção da Saúde (LIIPS/UNICESUMAR). Para tanto, os adolescentes, pais ou responsáveis foram instruídos quanto aos objetivos da pesquisa e foram sanadas eventuais dúvidas que poderiam ocorrer durante 0 preenchimento dos termos e questionário. Os participantes assinaram o termo de assentimento e os pais ou responsáveis assinaram o termo de consentimento livre e esclarecido (TCLE). Ressalta-se que o estudo seguiu todas as recomendações propostas na resolução 466/2012 do Ministério da Saúde, assim como a declaração de Helsinque.

Caracterização do estudo

O presente estudo caracteriza-se prospectivo, transversal e descritivo (THOMAS et al., 2012).

\section{Amostra do estudo}

Participaram do estudo $n=44$ adolescentes, sendo 22 do sexo masculino e 22 do sexo feminino.

\section{Critérios de inclusão e exclusão}

Como critério de inclusão, foram aceitos adolescentes com as seguintes características: a) ter entre 13 e 17 anos de idade, b) estar com excesso de peso ou obesidade dentro dos limites de corte propostos por Cole e Lobstein (2012), e c) estarem disponível para preencher o instrumento PedsQl - 4.0. Os critérios de 
exclusão foram: a) não ter o consentimento dos pais para preencher o inventário pediátrico, b) não ser classificado com excesso de peso ou obesidade em conformidade aos autores supramencionados e c) 0 não ter preenchido corretamente o questionário.

\section{O instrumento}

O PedsQL ${ }^{T M}$ foi desenvolvido com o propósito de analisar a qualidade de vida relacionada à saúde em crianças e adolescentes de 5-18 anos de idade, bem como outro questionário para os pais de crianças e adolescentes de 2-18 anos, sendo possível utilizá-lo em pacientes com distúrbios de saúde crônicos ou em crianças e adolescentes saudáveis (SOUZA et al., 2014).

O presente questionário foi traduzido e validado para a língua brasileira engloba 23 itens que abordam as seguintes dimensões: domínio físico (8 itens), domínio emocional (5 itens), domínio social (5 itens) e domínio escolar (5 itens), sendo desenvolvidos a partir de grupos de discussão, entrevistas cognitivas e prétestes (VARNI et al., 2001). A avaliação das crianças compreende as seguintes faixas etárias: 5-7, 8-12 e 13-18 anos de idade. De outra forma, o questionário dos pais contém as faixas etárias de: 2-4 anos para o pré-escolar, 5-7 para a criança pequena, 8-12 para a criança e 13-18 anos para o adolescente. Como respaldo, os itens para cada um dos questionários são equivalentes, diferindo-se somente à linguagem adequada ao nível de desenvolvimento e ao uso da primeira ou terceira pessoa (KLATCHOIAN et al., 2008). Para a aplicação, são necessários aproximadamente cinco minutos (VARNI et al., 2001). As questões interpelam o indivíduo quanto cada item representou um problema no último mês, com uma escala de respostas de cinco alternativas: $(0$ - nunca é um problema; 1 - quase nunca é um problema; 2 - algumas vezes é um problema; 3 - frequentemente é problema; 4 - quase sempre é um problema).

Ademais, a versão utilizada no mencionado estudo foi de 13-17 anos de idade. Em adição, o instrumento apresenta dois formatos para serem utilizados: um para o adolescente e outro para os pais. Outrossim, a verificação das respostas do PedsQL 4.0 ocorreram da seguinte forma: foram pontuados inversamente e transpostos linearmente para uma escala de 0-100 (nunca $=0=100$ pontos; quase nunca $=1=75$ pontos; algumas vezes $=2=50$ pontos, muitas vezes $=3=25$ pontos; quase sempre $=4=0$ pontos); assim, quanto maior o escore, melhor a QVRS.

O quadro 1 apresenta as questões que integram cada domínio utilizado na aplicação do instrumento.

QUADRO 1 - Descrição dos itens que compõem cada domínio do instrumento PedsQL $-4.0^{\mathrm{TM}}$.

\begin{tabular}{|ll|}
\hline Domínio físico & Domínio emocional \\
\hline A) Problemas quanto a minha saúde e atividade & B) Problemas quanto aos meus sentimentos \\
Dificuldade: Andar mais de um quarteirão & Dificuldade: Sentimento de medo ou assustado \\
Dificuldade: Realizar alguma corrida pela rua & Dificuldade: Sentimento de medo ou deprimido \\
Dificuldade: Praticar atividades esportivas & Dificuldade: Sentimento de raiva ou nervoso \\
Dificuldade: Levantar peso & Dificuldade: Dificuldade para dormir
\end{tabular}


Dificuldade: Tomar banho sozinho

Dificuldade: Auxiliar nas tarefas de casa

Dificuldade: Dor

Dificuldade: Pouca energia ou disposição

Domínio social

C) Problemas de relação com outras pessoas

Dificuldade: Convivência com outros adolescentes

Dificuldade: Problema para fazer amizade

Dificuldade: Provocação por parte de outros adolescentes

Dificuldade: Fazer coisas com outros adolescentes

Dificuldade: Acompanhar adolescentes da mesma idade

Nota: Os dados são expressos pela relação dos quatros domínios que englobam o instrumento: domínio físico, domínio emocional, domínio social e domínio escolar.

Os questionários foram respondidos no LIIPS/UNICESUMAR. Após o preenchimento, os dados foram conferidos e tabulados para posterior análise. Os adolescentes que preencheram menos de $50 \%$ do questionário foram excluídos das análises estatísticas.

\section{Analise estatística}

A normalidade da distribuição dos dados foi verificada para todas as respostas do questionário, por meio do teste de Kolmogorov-Smirnov. Mediante a confirmação, os dados foram expressos pela média e $( \pm)$ desvio padrão. Ademais, utilizou-se o teste $t$ de Student para amostras independentes com o propósito de comparar os sexos, assumindo um nível de significância de $5 \%$. As análises foram desempenhadas utilizando o pacote estatístico SPSS versão 20.0 (IBM, Estados Unidos da América).

\section{RESULTADOS E DISCUSSÃO}

Os resultados obtidos das avaliações dos escores do PedsQL 4.0 dos adolescentes participantes do estudo são apresentados na Tabela 1.

TABELA 1 - Escores do inventário pediátrico de qualidade de vida (PedsQL - 4.0 $0^{\mathrm{TM}}$ ) em adolescentes de ambos os sexos com excesso de peso e obesidade.

\begin{tabular}{ccccc}
\hline Domínios & Masculino & Feminino & Geral & Valor $\boldsymbol{p}$ \\
\hline Desempenho físico & $4,8 \pm 3,7$ & $5,9 \pm 3,5$ & $6,1 \pm 4,1$ & $p=0,1$ \\
Desempenho emocional & $5,9 \pm 3,6$ & $7,5 \pm 4,0$ & $7,0 \pm 4,2$ & $p=0,1$ \\
Desempenho social & $3,6 \pm 3,0$ & $3,6 \pm 3,7$ & $3,9 \pm 3,3$ & $p=0,9$ \\
Desempenho escolar & $5,3 \pm 3,5$ & $4,9 \pm 3,5$ & $5,5 \pm 3,5$ & $p=0,7$ \\
Somatória dos domínios & $20,4 \pm 11,0$ & $21,8 \pm 12,4$ & $20,8 \pm 11,7$ & $p=0,8$ \\
\hline
\end{tabular}

Nota: os dados são expressos pela média ( \pm ) desvio padrão. 
Conforme os resultados apresentados na Tabela 1, não foram verificadas diferenças significativas entre os diferentes domínios de ambos os sexos avaliados $(p>0,05)$. Os achados do presente estudo apontam baixa percepção de QVRS pelos adolescentes com excesso de peso ou obesidade (WHO, 2018). Sobre essa perspectiva, estudos conduzidos em países desenvolvidos, bem como países em desenvolvimento, como é o caso do Brasil, apresentaram resultados similares encontrados na presente pesquisa (BUTTITTA et al., 2014; GUIMARÃES et al., 2015; JALALI-FARAHAN et al., 2016).

Além do impacto global na QVRS (menor escore geral no PedsQL $-4.0^{\mathrm{TM}}$ ), a obesidade em crianças com excesso de peso ou obesidade atinge diferentes escalas de intensidade, em todos os domínios do inventário pediátrico: físico, social, emocional e escolar. A medida da QVRS caracteriza-se por ser um método empregado para avaliar a função física e psicossocial global (NORONHA et al., 2016). Em vista disso, instrumentos genéricos não são projetados para explorar todos os domínios que especificamente podem ser relacionados à obesidade. Entretanto, os mesmos podem apresentar um quadro global de como o indivíduo obeso percebe sua respectiva saúde e bem-estar (TESTA; SIMONSON, 1996).

No presente estudo, tanto para o sexo masculino quanto para o feminino, o domínio emocional e físico foram os mais congruentes à atenuação na QVRS entre os sexos. Em outra recente revisão, os achados indicaram resultados discrepantes, quando comparado a presente pesquisa, apresentando os domínios social e físico mais afetados (BUTTITTA et al., 2014). Fato este que possivelmente se justifica pelas alterações decorrentes do funcionamento físico exercidas pela obesidade na infância e subsequentemente, na adolescência. Implicações físicas, tais como: dificuldades na realização de atividades corriqueiras, como andar pela casa, tomar banho, assim como a consumação de outras práticas esportivas (CASTRO et al., 2016) podem impactar diretamente no domínio físico dos adolescentes. A supervalorização da mídia pelo peso corporal "ideal" e imagem corporal também estão associadas a fatores que influenciam a QVRS, sobretudo entre as meninas, bem como fatores físicos como a puberdade, hormônios e padrões sociais e de beleza, que são complexos de atingir (APARíCIO et al., 2016).

Por sua vez, evidências prévias identificaram singularidades entre meninos e meninas, sendo que o sexo feminino tende a responder distintamente de meninos, no que diz respeito ao padrão de beleza imposto pela sociedade. Nesse ponto de vista, o estudo de Pogodina et al. (2017) identificou que as meninas com excesso de peso ou obesidade necessitam de maior atenção, devido aos fatores intrínsecos impostos pela mídia, como o padrão de beleza feminina determinado pela mídia, como por exemplo o "culto ao corpo perfeito", visando apenas os componentes estéticos. Desse modo, considerando os fatores supramencionados, tornam-se imprescindíveis as intervenções de educação em saúde, que enquadrem as distinções entre meninos e meninas, para serem de fato, efetivas.

Por outro lado, destaca-se que nem todas as crianças com excesso de peso ou obesidade possuem obrigatoriamente limitações referentes aos domínios avaliados e com associação aos fatores de risco para as doenças crônicas nãotransmissíveis. Um ponto substancialmente positivo do PedsQL é apresentar duas versões, uma para os pais e outra para as crianças ou adolescentes (VARNI et al., 2001). Assim, torna-se possível utilizar o instrumento de acordo com as distinções entre cada faixa etária, ao utilizar uma linguagem mais assertiva. Destaca-se que o respectivo instrumento foi proposto para ser autoaplicável (VARNI et al., 1999). Portanto, justifica-se a adequação da linguagem em diferentes faixas etárias. No 
entanto, o estudo de validação para o Brasil demonstrou a possibilidade de ser administrado pelo entrevistador. Salienta-se ainda que a aplicação do instrumento é considerada rápida e fácil, apresentando a possibilidade do cálculo dos escores sumários e das escalas (KLATCHOIAN et al., 2008).

Em contrapartida, o PedsQL 4.0 como parâmetro dos diferentes domínios abordados, não deve ser exclusivamente um instrumento de diagnóstico da qualidade de vida. Consequentemente, outros mecanismos aplicáveis podem ser de grande valência quando utilizados isoladamente e/ou concomitantemente com 0 propósito de identificar a qualidade de vida dos adolescentes. Além disso, quando aplicado o instrumento em crianças dentro das faixas de corte estabelecidas como normais, para o peso corporal, outro estudo identificou que crianças saudáveis apresentaram escores significativamente maiores no domínio escolar, quando comparado a crianças com excesso de peso ou obesidade (CHAN; WANG, 2013). Em virtude disso, corrobora-se com os pontos supracitados, em que Kunkel et al. (2009) realizaram investigações com crianças obesas e não obesas e verificaram que o grupo com excesso de peso obteve escores inferiores de QVRS, quando comparado ao grupo sem excesso de peso, exceto para o domínio emocional dos adolescentes e na saúde psicossocial, para os pais.

Do mesmo modo, outra evidência identificou que a chance de um adolescente com excesso de peso ter baixa qualidade de vida foi 3,54 vezes (IC95\% = 1,94-6,47) maior que um adolescente sem excesso de peso, após os ajustes matemáticos (KUNKEL et al., 2009). Além de tudo, foram verificados que a QVRS foi significativamente menor em adolescentes com excesso de peso quando comparado aos adolescentes dentro dos padrões de normalidade para o peso corporal. Fator que ratifica as respostas encontradas por Kolotkin et al. (2018) que mostraram associação entre a melhora da QVRS, após a perda de peso corporal.

Frente a isso, a medida da QVRS pode ajudar os profissionais de saúde a investigar com melhor compreensão acerca de fatores nocivos à saúde de crianças e adolescentes com excesso de peso ou obesidade, e assim, promover ações preventivas visando a melhoria dos aspectos correlacionados a qualidade de vida (BUTTITTA et al., 2014; CHEN et al., 2014; GUIMARÃES et al., 2015; JALALIFARAHAN et al., 2016). Finalmente, a avaliação da QVRS em crianças obesas torna-se um parâmetro indispensável para avaliação do sucesso do tratamento da obesidade (CHAN; WANG, 2013).

\section{CONCLUSÃO}

Como resultado, considera-se uma forte relação entre a obesidade e baixa percepção de qualidade de vida relacionado à saúde dos adolescentes. Como complemento, os resultados pressupõem ainda, relativas proporções de adolescentes que estejam vivendo com menores índices de QVRS, por causa do excesso de peso e obesidade. Por outro lado, vale ressaltar, que o estudo não avaliou as características socioeconômicas dos participantes da pesquisa, tendo em vista que o nível socioeconômico pode interferir também nas respostas da QVRS dos adolescentes. Por fim, programas interdisciplinares de intervenção em promoção da saúde realizados de forma sensível e solidária, podem contribuir satisfatoriamente na melhoria da QVRS em adolescentes obesos. 


\section{AGRADECIMENTOS}

Centro universitário de Maringá - Unicesumar pelo suporte financeiro. Ao Grupo de Estudos em Educação Física, Fisioterapia, Esporte, Nutrição e Desempenho (GEFFEND/UniCesumar) e ao Instituto Cesumar de Ciência, Tecnologia e Inovação $-($ ICETI).

\section{REFERÊNCIAS}

APARÍCIO, E.; CANALS J.; ARIJA V.; HENAUW S.; MICHELS N. The role of emotion regulation in childhood obesity: implications for prevention and treatment. Nutrition Research Reviewes, v. 29, n. 1, p. 7-29, 2016. Disponível em: <https://www.researchgate.net/journal/1475-2700_Nutrition_Research_Reviews >. DOI: 10.1017/S095442241500015. Acesso em: 12/03/ $201 \overline{9}$.

BRANCO, L. M.; HILÁRIO, M. O. E.; CINTRA, I. P. Percepção e satisfação corporal em adolescentes e a relação com seu estado nutricional. Revista de Psiquiatria Clínica, v. $36, \quad$ n. 6, p. 292-296, 2006. Disponível em: <http://dx.doi.org/10.1590/S0101-60832006000600001>. DOI: 10.1590/S010160832006000600001. Acesso em: 12/03/2019.

BRANCO, L. M.; CARVALHO, I. Z.; GARCIA O. H.; FANHANI, A. P.; MACHADO S. M. C. et al. Effects of 2 Types of Resistance Training Models on Obese AdolescentsBody Composition, Cardiometabolic Risk, and Physical Fitness. Journal of Strength and Conditioning Research, v. 00, p. 1, 2018. [ahead of pub] <https://journals.Iww.com/nscajscr/Abstract/publishahead/Effects_of_2_Types_of_Re sistance Training Models.95035.aspx.>. DOI: 10.1519 / JSC.0000000000002877 Acesso em: 02/04/2019.

BUTTITTA, M.; ILIESCU, C.; ROUSSEAU, A.; GUERRIEN, A. Quality of life in overweight and obese children and adolescents: A literature review. Quality of Life Research, v. 23, n. 4, p. 1117-1139, 2014. Disponível em: <https://www.ncbi.nlm.nih.gov/pubmed/24249217>. DOl:10.1007/s11136-013-05685. Acesso em: 12/032019.

CASTRO, G. G.; FIGUEIREDO G. L. A.; SILVA T. S.; FARIA K. C. Qualidade de vida em crianças escolares com sobrepeso e obesidade. Revista do Departamento de Educação Física e Saúde e do Mestrado em Promoção da Saúde da Universidade de Santa Cruz do Sul - Unisc, v. 17, n. 4, p. 287-291, 2016. Disponível em: https://online.unisc.br/seer/index.php/cinergis/article/view/8067/5345> DOI: http://dx.doi.org/10.17058/cinergis.v17i3.8067. Acesso em: 01/04/2019.

CHAN, C. M.; WANG, W. C. Quality of life in overweight and obese young Chinese children: a mixed-method study. Health and Quality of Life Outcomes, v. 11, n. 33, p. 33-43, 2013 Disponível em: <https://www.ncbi.nlm.nih.gov/pubmed/23496917>. Acesso em: 15/03/2019.

CHEN, G.; RATCLIFFE, J.; OLDS, T.; MAGAREY, A.; JONES, M. et al. BMI health behaviors and quality of life in children and adolescents: a school-based study. Pediatrics, v.133, p.868-874, 2014. Disponível em: <http://pediatrics.aappublications.org/content/early/2014/02/25/peds.2013-0622>. Acesso em: 10/03/2019. 
COLE, T. J; LOBSTEIN, T. Extended international (IOTF) body mass index cut-offs for thinness, overweight and obesity. Pediatric Obesity, p.284-294, 2012. Disponível em: <https://www.ncbi.nlm.nih.gov/pubmed/22715120>. DOI: 10.1111/j.2047-6310.2012.00064. Acesso em: 10/03/2019.

CUNHA, L. M.; PANTOJA, M. S.; LIMA, A. V. M.; PORTELLA, M. B.; FURLANETO, I. $P$. Impacto negativo da obesidade sobre a qualidade de vida de crianças. Revista Brasileira de Obesidade, Nutrição e Emagrecimento, v. 12, n. 70, p. 231-238, mar./abril. $2018 . \quad$ Disponível em: <http://www.rbone.com.br/index.php/rbone/article/view/686>. DOI: $1981-9919$. Acesso em: 10/03/2019.

GOUVEIA, M. J.; FRONTINI, R.; CANAVARRO, M. C.; MOREIRA, H. Quality of life and psychological functioning in pediatric obesity: the role of body image dissatisfaction between girls and boys of different ages. Quality of Life Research, v. 23, n. 9, p. 2629-2238, 2014. Disponível em: <https://www.ncbi.nlm.nih.gov/pubmed/24817248>. DOI: 10.1007/s11136-014-0711y. Acesso em: 11/03/2019.

GUIMARÃES, M. A. P.; QUADROS JUNIOR, M. C.; FONSECA, M. A.; AMORIM C. R.; PINTO, E. P. Características socioeconômicas, prática de atividade física e qualidade de vida de escolares da rede pública. Revista Arquivos de Ciências da Saúde, v. 22, n. 9, p. 57-62, 2015. Disponível em: <https://doi.org/10.17696/23183691.22.2.2015.144>. DOI: 10.17696/2318-3691.22.2.2015.144. Acesso em: $11 / 03 / 2019$.

JALALI-FARAHANI, S.; ALAMDARI, S.; KARIMI M.; AMIRI, P. Is overweight associated with health related quality of life (HRQoL) among Tehranian school children? Springer Plus, v. 5, p. 313, 2016. Disponível em: <https://springerplus.springeropen.com/articles/10.1186/s40064-016-1930-1> DOI: 10.1186 / s40064-016-1930-1. Acesso em: 10/03/2019.

KLATCHOIAN, D. A.; LEN, C. A.; TERRERI, M. T.; SILVA, M.; ITAMOTO, M. S.; CICONELLI, R. M. Quality of life of children and adolescents from São Paulo: reliability and validity of the Brazilian version of the Pediatric Quality of Life Inventory version 4.0 Generic Core Scales. The Journal of Pediatrics, v. 84, n. 9, p. 308-315, 2008. Disponível em: <http://dx.doi.org/10.1590/S0021-75572008000400005>. DOI: 10.1590/S0021-75572008000400005. Acesso em:10/03/2019.

KOLOTKIN, R. L.; ANDERSEN, J. R. A systematic review of reviews: exploring the relationship between obesity, weight loss and health-related quality of life. Clinical Obesity, v. 7, n. 5, p. 273-289, 2017. Disponível em:< https://www.ncbi.nlm.nih.gov/pmc/articles/PMC5600094/. DOI: 10.1111/ cob.12203>. Acesso em: 10/03/2019.

KOLOTKIN, R. L.; GABRIEL SMOLARZ, B.; MEINCKE, H. H.; FUJIOKA, K. Improvements in health-related quality of life over 3 years with liraglutide $3.0 \mathrm{mg}$ compared with placebo in participants with overweight or obesity. Clinical Obesity, v. $8, \quad$ n. $1, \quad$ p. 1-10, 2018 . Disponível em: 
<https://www.ncbi.nlm.nih.gov/pmc/articles/PMC5813214/>. $\quad$ DOI: 10.1111 / cob.12226. Acesso em:10/03/2019.

KUNKEL, N.; OLIVEIRA, W. F.; PERES, M. A. Excesso de peso e qualidade de Vida relacionada à saúde em adolescentes de Florianópolis -SC. Revista de Saúde Pública, v. 43, n. 2, p. 226-235, 2009. Disponível em: $<$ http://www.scielo.br/scielo.php?pid=S003489102009000200003\&script=sci_abstrac t\&tlng=pt>. Acesso em: 10/03/2019.

LA SCALA, C. S. K.; NASPITZ, C. K.; SOLÉ, D. Adaptation and validation of the Pediatric Asthma Quality of Life Questionnaire (PAQLQ) in Brazilian asthmatic children and adolescents. Journal of Pediatric, v. 81, n. 1, p. 54-60, 2005. Disponível em: <http://dx.doi.org/10.1590/S0021-75572005000100011>. DOI: 10.1590/S0021-75572005000100011. Acesso em: 11/03/2019.

MULLER, R. C. L. Obesidade na adolescência. Revista Brasileira de Medicina, v. 37, n. 1, p. 45-48, 2001.Disponível em: $<$ Http://www.moreirajr.com.br/revistas.asp?fase=r003\&id_materia=1413>. Acesso em: 11/03/2019.

NARDO JÚNIOR, N.; BIANCHINI, J. A. A.; SILVA, D. F.; FERRARO, Z. M.; LOPERA, C. A. et al. Building a response criterion for pediatric multidisciplinary obesity intervention success based on combined benefits. European Journal, v. 1, n. 6, p. 1-12, 2018. <https://link.springer.com/article/10.1007/s00431-018-31150\#citeas >. DOI: 10.1007 / s00431-018-3115-0. Acesso em: 02/04/2019.

NORONHA D. D.; MARTINS, A. MARIA E. B. L.; DIAS, D. S.; SILVEIRA, M. F.; PAULA, A. M. B. et al. Qualidade de vida relacionada à saúde entre adultos e fatores associados: um estudo de base populacional. Ciência \& Saúde Coletiva, v. 21, n. 2, p. 463-474, 2016. Disponível em: <http://www.scielo.br/pdf/csc/v21n2/1413-8123csc-21-02-0463.pdf>. DOI: 10.1590/1413-81232015212.01102015. Acesso em: $11 / 03 / 2019$.

PANTOJA, M. S.; PORTELLA, M. B.; CUNHA, L. M.; LIMA, A. V. M.; FURLANETO, I. $P$. Qualidade de vida relacionada à saúde de crianças obesas. Revista Brasileira de Qualidade de Vida, v. 9, n. 4, p. 283-297, out./dez. 2017. Disponível em: <https://periodicos.utfpr.edu.br/rbqv/article/view/6939/4575>. DOI:

10.3895/rbqv.v9n4.6939. Acesso em: 29/03/2019.

PEROSA, G. B.; AMATO, I. A.; RUGOLO, L. M. S. S.; FERRARI, G. F.; OLIVEIRA, M. C. F. A. Quality of life of asthmatic children and adolescents: relation to maternal coping. Revista Paulista de Pediatria, v. 31, n. 2, p. 145-151, 2013. Disponível em: <http://dx.doi.org/10.1590/S0103-05822013000200003>. DOI: $10.1590 / S 0103-$ 05822013000200003. Acesso em: 11/03/2019.

POGODINA, A.; RYCHKOVA L.; KRAVTZOVA, O.; KLIMKINA J.; KOSOVTZEVA A. Cardiometabolic risk factors and health-related quality of life in adolescents with obesity. Journal of Childhood Obesity, v. 13, n. 6, p. 499-506, 2017. Disponível em: <https://doi.org/10.1089/chi.2016.0330>. DOI: 10.1089/chi.2016.0330. Acesso em: 29/03/2019. 
ROCHA, M.; PEREIRA, H.; MAIA, R.; SILVA, E.; MORAIS, N. et al. Aspectos psicossociais da obesidade na infância e adolescência. PSICOLOGIA, SAUDE \& DOENÇAS, Lisboa, v. 18, n. 3, p. 713-723, dez. 2017. Disponível em:<http://www.scielo.mec.pt/scielo.php?script=sci_arttext\&pid=S1645-

00862017000300007\&lng=pt\&nrm=iso $>$. DOI: 10.15309/17psd1807. Acesso em: 01/04/2019.

SILVA, K.; SILVA, F. M.; AGRÍCOLA, P. M. D.; NETO, I. O. Imagem Corporal Em Adolescentes Obesas: Uma Revisão. Revista Brasileira de Obesidade, Nutrição e $\begin{array}{llllll}\text { emagrecimento, } & \text { v. } 11, \quad \text { n. } & 64, \quad \text { p. } & 217-223,\end{array}$ <file:///C:/Users/Alunos.DP29748/Downloads/522-2354-1-PB\%20(1).pdf> Acesso em: 10/03/2019.

SOUZA, J. G. S.; PAMPONET, M. A.; SOUZA, T. C. S.; PEREIRA, A. R.; SOUZA, A. G. S. et al. Instrumentos utilizados na avaliação da qualidade de vida de crianças brasileiras. Revista Paulista de Pediatria, v. 32, n. 2, p. 272-278, 2014. Disponível em: $\quad$ http://www.scielo.br/pdf/rpp/v32n2/0103-0582-rpp-32-02-00272.pdf>. DOI: 10.1590/0103-0582201432214313. Acesso em: 11/03/2019.

TESTA, M. T.; SIMONSON D. C. A. Assessment of quality of life outcomes. New England Journal of Medicine, v. 28, n.13, p. 835-840, 1996. Disponível em: $<$ https://www.ncbi.nlm.nih.gov/pubmed/8596551>.DOI:

10.1056/NEJM199603283341306. Acesso em: 29/03/2019.

THOMAS, J. R.; NELSON, J. K.; SILVERMAN S. J. Métodos de pesquisa em atividade física, 5. ed. Porto Alegre: Artmed: 2012. Acesso em: 17/03/2019.

VARNI, J. W.; SEID M.; KURTIN P. S. The PedsQL TM 4.0: Reliability and validity of the pediatric quality of life inventory version 4.0: generic core scales in healthy and patient populations. Medical Care, v. 39, n. 8, p. 800-912, 2001. Disponível em: <https://www.ncbi.nlm.nih.gov/pubmed/11468499>. Acesso em: 29/03/2019.

VARNI, J. W.; SEID, M.; RODE, C. A. The PedsQL: measurement model for the pediatric quality of life inventory. Medical Care, v. 37, n. 2, p.126-139, 1999. Disponível em: <https://www.ncbi.nlm.nih.gov/pubmed/10024117>. Acesso em: 29/03/2019.

WORLD HEALTH ORGANIZATION. Obesity: preventing and managing the global epidemic. Geneve: World Health Organization, 2018. Acesso em: 10/01/2019.

PEDIATRIC QUALITY OF LIFE INVENTORY - PedsQLTM 4.0

No ÚLTIMO MÊS, quão problemático tem sido para você?

\begin{tabular}{|l|l|l|l|l|l|}
\hline $\begin{array}{l}\text { PROBLEMAS QUANTO Ȧ MINHA SAÚDE E } \\
\text { ATIVIDADES }\end{array}$ & Nunca & $\begin{array}{l}\text { Quase } \\
\text { Nunca }\end{array}$ & $\begin{array}{l}\text { Algumas } \\
\text { vezes }\end{array}$ & $\begin{array}{l}\text { Muitas } \\
\text { Vezes }\end{array}$ & $\begin{array}{l}\text { Quase } \\
\text { Sempre }\end{array}$ \\
\hline 1. Para mim é difícil andar mais de um quarteirão & & & & & \\
\hline 2. Para mim é difícil correr & & & & & \\
\hline $\begin{array}{l}\text { 3. Para mim é difícil praticar esportes ou fazer } \\
\text { exercícios físicos }\end{array}$ & & & & & \\
\hline 4. Para mim é difícil levantar coisas pesadas & & & & & \\
\hline $\begin{array}{l}\text { 5. Para mim é difícil tomar banho de banheira ou } \\
\text { de chuveiro sozinho }\end{array}$ & & & & & \\
\hline 6. Para mim é difícil ajudar nas tarefas domésticas & & & & & \\
\hline
\end{tabular}

ENCICLOPÉDIA BIOSFERA, Centro Científico Conhecer - Goiânia, v.16 n.29; p. 2012 


\begin{tabular}{|l|l|l|l|l|l|}
\hline 7. Eu sinto dor & & & & & \\
\hline 8. Eu tenho pouca energia ou disposição & & & & & \\
\hline
\end{tabular}

\begin{tabular}{|l|l|l|l|l|l|}
\hline $\begin{array}{l}\text { PROBLEMAS QUANTO AOS MEUS } \\
\text { SENTIMENTOS }\end{array}$ & Nunca & $\begin{array}{l}\text { Quase } \\
\text { Nunca }\end{array}$ & $\begin{array}{l}\text { Algumas } \\
\text { vezes }\end{array}$ & $\begin{array}{l}\text { Muitas } \\
\text { Vezes }\end{array}$ & $\begin{array}{l}\text { Quase } \\
\text { Sempre }\end{array}$ \\
\hline 1. Eu sinto medo ou assustado & & & & & \\
\hline 2. Eu me sinto triste ou deprimido & & & & & \\
\hline 3. Eu sinto raiva (zangado) & & & & & \\
\hline 4. Eu tenho problemas para dormir & & & & & \\
\hline $\begin{array}{l}\text { 5. Eu me preocupo com o que vai acontecer } \\
\text { comigo }\end{array}$ & & & & \\
\hline
\end{tabular}

\begin{tabular}{|l|l|l|l|l|l|}
\hline $\begin{array}{l}\text { PROBLEMAS EM COMO EU ME } \\
\text { RELACIONO COM OUTRAS PESSOAS }\end{array}$ & Nunca & $\begin{array}{l}\text { Quase } \\
\text { Nunca }\end{array}$ & $\begin{array}{l}\text { Algumas } \\
\text { vezes }\end{array}$ & $\begin{array}{l}\text { Muitas } \\
\text { Vezes }\end{array}$ & $\begin{array}{l}\text { Quase } \\
\text { Sempre }\end{array}$ \\
\hline $\begin{array}{l}\text { 1. Eu tenho dificuldade para conviver com outros / } \\
\text { outras adolescentes }\end{array}$ & & & & & \\
\hline $\begin{array}{l}\text { 2. Os (as) outros (as) adolescentes não querem } \\
\text { ser meus amigos (as) }\end{array}$ & & & & & \\
\hline $\begin{array}{l}\text { 3. Os (as) outros (as) adolescentes implicam } \\
\text { comigo }\end{array}$ & & & & & \\
\hline $\begin{array}{l}\text { 4. Eu não consigo fazer coisas que outros (as) } \\
\text { adolescentes da minha idade fazem }\end{array}$ & & & & & \\
\hline $\begin{array}{l}\text { 5. Para mim é difícil acompanhar os (as) } \\
\text { adolescentes da minha idade }\end{array}$ & & & & & \\
\hline
\end{tabular}

\begin{tabular}{|l|l|l|l|l|l|}
\hline PROBLEMAS QUANTO À ESCOLA & Nunca & $\begin{array}{l}\text { Quase } \\
\text { Nunca }\end{array}$ & $\begin{array}{l}\text { Algumas } \\
\text { vezes }\end{array}$ & $\begin{array}{l}\text { Muitas } \\
\text { Vezes }\end{array}$ & $\begin{array}{l}\text { Quase } \\
\text { Sempre }\end{array}$ \\
\hline 1. É difícil prestar atenção na aula & & & & & \\
\hline 2. Eu esqueço as coisas & & & & & \\
\hline $\begin{array}{l}\text { 3. Eu tenho dificuldade para acompanhar a minha } \\
\text { nas tarefas escolares }\end{array}$ & & & & & \\
\hline 4. Eu falto à aula por não estar me sentindo bem & & & & & \\
\hline 5. Eu falto à aula para ir ao médico ou ao hospital & & & & & \\
\hline
\end{tabular}

